\title{
Erratum to: Two Optimal Inequalities for Anti-holomorphic Submanifolds and Their Applications
}

\author{
Falleh R. Al-Solamy, Bang-Yen Chen* and Sharief Deshmukh
}

\begin{abstract}
Theorem 4.1 of [1] is not correctly stated. In this erratum we make a correction on Theorem 4.1. As a consequence, we also make the corresponding correction on Theorem 5.2 of [1].
\end{abstract}

We follow the notation from the original article [1]. Theorem 4.1 of [1] is not correctly stated. The correct statement shall read as follows.

Theorem 4.1. Let $N$ be an anti-holomorphic submanifold of a complex space form $\widetilde{M}^{h+p}(4 c)$ with $h=\operatorname{rank}_{\mathbf{C}} \mathcal{D} \geq 1$ and $p=\operatorname{rank} \mathcal{D}^{\perp} \geq 2$. Then we have

$$
\delta(\mathcal{D}) \leq \frac{(2 h+p)^{2}}{2} H^{2}+\frac{p}{2}(4 h+p-1) c-\frac{3 p^{2}}{2(p+2)}\left|H_{\mathcal{D}^{\perp}}\right|^{2} .
$$

The equality sign of (4.3) holds identically if and only if the following three conditions are satisfied:

(a) $N$ is $\mathcal{D}$-minimal, i.e., $\vec{H}_{\mathcal{D}}=0$,

(b) $N$ is mixed totally geodesic, and

(c) there exists an orthonormal frame $\left\{e_{2 h+1}, \ldots, e_{n}\right\}$ of $\mathcal{D}^{\perp}$ such that the second fundamental $\sigma$ of $N$ satisfies

$$
\begin{cases}\sigma_{r r}^{r}=3 \sigma_{s s}^{r} & \text { for } 2 h+1 \leq r \neq s \leq 2 h+p, \\ \sigma_{s t}^{r}=0 & \text { for distinct } r, s, t \in\{2 h+1, \ldots, 2 h+p\} .\end{cases}
$$

This correction shall be made since the last formula $(4.13)$ in the proof of Theorem 4.1 contains an error. The corrected (4.13) shall read as

$$
\begin{aligned}
& \frac{(2 h+p)^{2}}{2} H^{2}+\frac{p}{2}(4 h+p-1) c-\delta(\mathcal{D}) \\
\geq & 2 h^{2}\left|\vec{H}_{\mathcal{D}}\right|^{2}+\sum_{i=1}^{2 h} \sum_{r=2 h+1}^{2 h+p}\left\|\sigma\left(e_{i}, e_{r}\right)\right\|^{2}+\frac{3 p^{2}}{2(p+2)}\left|H_{\mathcal{D}^{\perp}}\right|^{2} \\
\geq & \frac{3 p^{2}}{2(p+2)}\left|H_{\mathcal{D}^{\perp}}\right|^{2} .
\end{aligned}
$$

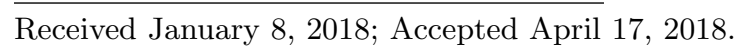

Communicated by Tai-Chia Lin.

2010 Mathematics Subject Classification. 53C40.

Key words and phrases. anti-holomorphic submanifolds, optimal inequality, CR $\delta$-invariant.

*Corresponding author. 
Consequently, Theorem 5.2 in [1] shall be replaced by

Theorem 5.2. Let $N$ be an anti-holomorphic submanifold in a complex space form $\widetilde{M}^{1+p}(4 c)$ with $h=\operatorname{rank}_{\mathbf{C}} \mathcal{D}=1$ and $p=\operatorname{rank} \mathcal{D}^{\perp} \geq 2$. Then we have

$$
\delta(\mathcal{D}) \leq \frac{(p+2)^{2}}{2} H^{2}+\frac{p(p+3)}{2} c-\frac{3 p^{2}}{2(p+2)}\left|H_{\mathcal{D}^{\perp}}\right|^{2} .
$$

The equality case of (5.4) holds identically if and only if $c=0$ and either

(i) $N$ is a totally geodesic anti-holomorphic submanifold of $\mathbf{C}^{h+p}$ or,

(ii) up to dilations and rigid motions, $N$ is given by an open portion of the following product immersion:

$$
\phi: \mathbf{C} \times S^{p}(1) \rightarrow \mathbf{C}^{1+p} ; \quad(z, x) \mapsto(z, w(x)), \quad z \in \mathbf{C}, x \in S^{p}(1),
$$

where $w: S^{p}(1) \rightarrow \mathbf{C}^{p}$ is the Whitney $p$-sphere.

\section{References}

[1] F. R. Al-Solamy, B.-Y. Chen and S. Deshmukh, Two optimal inequalities for antiholomorphic submanifolds and their applications, Taiwanese J. Math. 18 (2014), no. 1, $199-217$.

Falleh R. Al-Solamy

Department of Mathematics, King Abdulaziz University, Jeddah 21589, Saudi Arabia E-mail address: falleh@hotmail.com

\section{Bang-Yen Chen}

Department of Mathematics, Michigan State University, 619 Red Cedar Road, East

Lansing, MI 48824-1027, USA

E-mail address: chenb@msu.edu

Sharief Deshmukh

Department of Mathematics, King Saud University, Riyadh 11451, Saudi Arabia E-mail address: shariefd@ksu.edu.sa 